\title{
In vitro and in vivo properties of a fully human IgG1 monoclonal antibody that combats multidrug resistant Pseudomonas aeruginosa
}

\author{
AZMI ADAWI ${ }^{1}$, CARLO BISIGNANO $^{2}$, TIZIANA GENOVESE $^{3}$, ANGELA FILOCAMO $^{2}$, \\ CAMELLIA KHOURI-ASSI ${ }^{4}$, ANAT NEVILLE ${ }^{5}$, GIORA Z. FEUERSTEIN ${ }^{6}$, \\ SALVATORE CUZZOCREA $^{3}$ and LEWIS F. NEVILLE ${ }^{1}$
}

\author{
${ }^{1}$ Lostam BioPharmaceuticals, Nazareth, Israel; ${ }^{2}$ Department of Pharmacobiology, School of Pharmacy and \\ ${ }^{3}$ Department of Clinical and Experimental Medicine and Pharmacology, School of Medicine, University of Messina, \\ Messina, Italy; ${ }^{4}$ St. Vincent Hospital, Nazareth; ${ }^{5}$ Department of Family Medicine, Ben-Gurion University of the Negev, \\ Beer-Sheva, Israel; ${ }^{6}$ Farmacon Consulting, Bryn Mawr, PA, USA
}

Received May 1,2012; Accepted May 31, 2012

DOI: $10.3892 / \mathrm{ijmm} .2012 .1040$

\begin{abstract}
The development of an anti-bacterial drug in the form of a monoclonal antibody (mAb) targeting an exposed virulence factor, represents an innovative therapeutic strategy. Consequently, a fully human IgG1 mAb (LST-007) targeting Pseudomonas aeruginosa (PA) flagellin type b was recombinantly expressed and characterized in vitro and in an infection model driven by a multidrug resistant (MDR) PA strain. LST-007 demonstrated a highly specific binding towards whole PA bacteria harboring flagellin type $b$ and its recombinant counterpart, with a $\mathrm{K}_{\mathrm{D}}$ of $7.4 \times 10^{-10} \mathrm{M}$. In bioactivity assays, LST-007 or titers of Cmax sera derived from pharmacokinetic studies, markedly attenuated PA motility in an equipotent manner. In vivo, parenteral LST-007 $(20 \mathrm{mg} / \mathrm{kg})$ given as a single or double-dosing paradigm post-infection, afforded survival (up to $75 \%$ at Day 7 ) in a lethal model of pneumonia driven by the intratracheal (i.t.) instillation of an $\mathrm{LD}_{80}$ of the MDR PA isolate. This protective effect was markedly superior to that of imipenem (30\% survival at Day 7) and totally devoid with an irrelevant, human isotype mAb. These data lay credence that LST-007 may be a valuable adjunct to the limited list of anti-bacterials that can tackle MDR PA strains, thereby warranting its continued development for eventual clinical evaluation.
\end{abstract}

\section{Introduction}

Regrettably, the emergence of multidrug resistant (MDR) strains of bacteria during the last 2-3 decades has failed to

Correspondence to: Dr Lewis F. Neville, Lostam BioPharmaceuticals Ltd., 13 Vadi Elhaj Street, Nazareth Illit 17111, Israel

E-mail: lewis@lostam-biopharma.com

Key words: Pseudomonas aeruginosa, multidrug resistance, monoclonal antibody, passive immunization, flagellin ignite intensive research and development efforts towards the discovery of novel antimicrobial drugs. This is exemplified by the fact that only 2 new classes of antibiotics have been marketed since 1970 (1,2). The Gram-negative bacterium Pseudomonas aeruginosa (PA) is an opportunistic pathogen and may be regarded as a nosocomial 'predator' especially in immunocompromised patients $(3,4)$. PA infections may cause life-threatening conditions such as ventilator-associated and hospital-acquired pneumonias as well as bloodstream and urinary tract infections in susceptible patients $(5,6)$. Additionally, PA plagues cystic fibrosis (CF) patients with at least $50 \%$ of the $\sim 60,000$ population in the US and EU permanently colonized with the bacterium $(7,8)$. Indeed, PA is a major cause of morbidity and mortality in these patients $(7,9)$. A remarkable feature of PA lies not only in its genomic diversity with a plethora of ORFs capable of degrading antibiotics (10) but also its capacity to acquire external genomic elements providing additional resistance mechanisms (11). Ultimately, MDR PA can be a grave problem, especially in the critical care setting where mortality rates for certain infections may range from 38 to $>70 \%$ (12). Thus, designing the appropriate antimicrobial therapy may be a clinical dilemma due to the paucity of effective and safe drugs that can combat MDR strains of PA.

An alternative and highly innovative anti-bacterial therapeutic approach apart from classical antibiotics is to disarm bacterial pathogens thereby augmenting the host's innate immune system to clear the infection (13). The notion of disarmament of bacterial pathogens by targeting virulence factors has allowed a resurgence in the importance and potential therapeutic role of anti-infective monoclonal antibodies (mAbs). Such advances have been catalyzed by a number of methodological breakthroughs which have permitted not only the rapid generation of fully human or humanized $\mathrm{mAb}$ candidates but also the capabilities to recombinantly express mammalian cell-derived mAbs at g/l quantities $(14,15)$. Pre-clinically, a number of pharmacological studies have demonstrated the effectiveness of anti-PA virulence mAbs which have justified their clinical evaluation (16-18). For 
example, KB001 and panobacumab, human mAbs targeting PA virulence factors PcrV and 011 LPS serotype, respectively, have demonstrated positive clinical findings, albeit in a limited number of hospital-associated pneumonia and CF cases when added to standard-of-care treatment $(19,20)$. These findings support passive immunization approaches targeting virulence factors especially since from an evolutionary point of view, they may be refractory to selection pressures due to their pivotal roles in bacterial infectivity and survival.

In accord with the above, we also believe that passive immunization with anti-PA virulence $\mathrm{mAbs}$ is a viable therapeutic proposition, representing an unfulfilled medical requirement. In contrast to the PcrV and 011 LPS virulence factors, our preferred PA target is the surface-expressed, single polar flagellum of which flagellin is the primary protein component $(21,22)$. Flagellar structures are pivotal for a number of PA's functions including bacterial motility, attachment and invasion to susceptible cells as well as being highly pro-inflammatory via the Toll-like receptor 5 (TLR5) (21-23). Consequently, a neutralizing $\mathrm{mAb}$ targeting the flagella of PA may intervene at a variety of critically important steps and curtail the catastrophic sequelae of events that lead to endorgan infection, dysregulated inflammation and mortality.

It should be noted that in PA, 2 types of flagellin have been identified and termed type a and type $b$ which may be discriminated on the basis of molecular size and reactions with type-specific polyclonal and mAbs $(24,25)$. In contrast to salmonella flagellins, PA flagellin types a and b do not exhibit phase variation since a single strain produces a single type of flagellin with no switching between types $a$ and $b$ (26). Numerous in vivo studies have pointed to the protective effects of either polyclonal antibodies or specific anti-flagellin mAbs following infection with antibiotic-sensitive PA strains harboring the appropriate, homologous flagellin type (27-31). Indeed, such antibody preparations provided equivalent protection to imipenem, a standard-of-care carbapenem antibiotic $(30,31)$. Since $>98 \%$ PA strains harbor a phenotypic type $\mathrm{a}$ or $\mathrm{b}$ flagellin $(24,27)$, an appropriate mAb therapy governed by a priori knowledge of the infecting flagellin type may permit a 'patient-tailored' anti-infective therapy and thus a higher probability of clinical success.

In the present study, we applied a battery of in vitro and in vivo tests to characterize our fully human, lead $\mathrm{mAb}$ termed LST-007 that targets PA flagellin type b. To our knowledge, this is the first report demonstrating that an antiinfective PA mAb administered as a monotherapy, can curb lethality driven by a clinically relevant MDR PA strain.

\section{Materials and methods}

Reagents. All general chemicals were purchased from Sigma (Rehovot, Israel) and HyLabs (Rehovot, Israel). Imipenem in the form of the marketed drug Tienam, was acquired from the Department of Pharmacy, University of Messina. Bacterial PA strains 27853, 25619 and 9721 were purchased from Microbiologics (USA). Ka02, a hospital-derived MDR PA strain, demonstrated sensitivity only to amikacin, colistin and partial sensitivity to gentamicin as determined from Vitek screens. LST-003, a proprietary chimeric $\mathrm{mAb}$ targeting PA flagellin type a was transiently expressed in $\mathrm{CHO}-\mathrm{K} 1$ cells and used in certain in vitro studies. A human IgG1 isotype control $\mathrm{mAb}(32)$ was used in a number of in vitro and in vivo studies.

Expression and purification of recombinant $P A$ flagellin type $b$. The fliC gene (Pa1092 from the PA genome database) corresponding to flagellin type $b$ of the laboratory strain PAO1, was used as the template for recombinant expression. This gene sequence was custom synthesized to permit appropriate codon usage for optimal expression in $E$. coli without modifying its primary amino acid sequence (Geneart, Germany). This cDNA sequence was engineered with 5' NdeI and $3^{\prime}$ HindIII restriction sites enabling direct ligation into a pET28a expression plasmid cleaved with these respective enzymes. The resultant recombinant protein harbored an $\mathrm{N}$-terminal histidine- 6 tag enabling immobilized metal affinity chromatography (IMAC) purification. The pET28a/ flagellin type $b$ recombinant plasmid was transformed into BL21 DE3 STAR cells (Invitrogen, Carlsbad, CA, USA) and a single colony was grown in $10 \mathrm{ml}$ LB containing $50 \mu \mathrm{g} / \mathrm{ml} \mathrm{kanamycin} \mathrm{overnight.} \mathrm{Thereafter,} 0.2 \mathrm{ml}$ of the overnight culture was taken to inoculate $50 \mathrm{ml}$ pre-warmed LB-kanamycin and at an OD of 0.3, IPTG was added to a final concentration of $1 \mathrm{mM}$ to induce expression for $3 \mathrm{~h}$ at $37^{\circ} \mathrm{C}$. Since the bulk of the recombinant protein was shown to reside in the insoluble fraction, induced bacterial pellets were resuspended in $50 \mathrm{mM}$ phosphate buffer ( $\mathrm{pH}$ 7.4) containing IGEPAL, sonicated and following centrifugation, the insoluble pellet was taken for IMAC as follows. The pellet was solubilized in buffer containing $6 \mathrm{M} \mathrm{GuHCl}$ and subsequently purified by IMAC (HiTrap chelating $5 \mathrm{ml}$ column charged with nickel). Recombinant PA flagellin type b was refolded on the column using a gradient 6-0 $\mathrm{M} \mathrm{GuHCl}$ and an elution was performed using an imidazole gradient (0-500 mM). Peak fractions were pooled, dialyzed against PBS, quantified using BCA and adjusted with PBS to a stock concentration of $1 \mathrm{mg} /$ $\mathrm{ml}$ and stored at $-20^{\circ} \mathrm{C}$. Coomassie gel staining demonstrated that the Mw of PA flagellin type b was $\sim 55 \mathrm{kDa}$ with $>95 \%$ purity. In small-scale expression and purification studies, recombinant PA flagellin type a was produced in an identical manner as described for flagellin type $b$ and yielded a protein of $\mathrm{Mw} \sim 45 \mathrm{kDa}$ with $>95 \%$ purity.

Expression and purification of LST-007. LST-007 was isolated from EBV-transformed human B cells (unpublished data) and its respective $\mathrm{V}_{\mathrm{H}}$ and $\mathrm{V}_{\mathrm{L}}$ cDNA chains were taken for expression using a commercially available, platform technology. In brief, following cloning and expansion of transduced LST-007 $\mathrm{V}_{\mathrm{H}}$ and LST-007 $\mathrm{V}_{\mathrm{L}}$ pools, shaker flasks of $\mathrm{CHO}$ cells supported in serum-free media were co-infected and clarified supernatant were taken for standard purification using a MabSelect medium. Purified LST-007 was concentrated in a stirred cell, dialyzed vs. phosphate buffer and quantitated at $280 \mathrm{~nm}$. Bioburden of sterile-filtered material was $0 \mathrm{cfu} / \mathrm{ml}$ with levels of endotoxin $<0.04 \mathrm{EU} / \mathrm{mg}$. Final concentration of LST-007 was quantified at $13.15 \mathrm{mg} / \mathrm{ml}$ and stored as a number of $1-\mathrm{ml}$ aliquots at -20 and $-80^{\circ} \mathrm{C}$.

Binding of LST-007 towards recombinant PA flagellin type $b$ in ELISA. Wells of Maxisorp ELISA plates (\#442404; Nunc Brand Products) were coated with $250 \mathrm{ng}$ of recombinant 
PA flagellin type $b$ and incubated with orbital shaking for $2 \mathrm{~h}$ at room temperature. Thereafter, unbound antigen was removed and $200 \mu \mathrm{l}$ blocking solution comprised of PBS-10\% fetal bovine serum (FBS; \#04-001-1A; Biological Industries) was added overnight at $4^{\circ} \mathrm{C}$. Following removal of blocking buffer, $50 \mu \mathrm{l}$ LST-007 in blocking solution was added over a range of $0-208 \mathrm{pM}(0-31.25 \mathrm{ng} / \mathrm{ml})$ and allowed to incubate for $2 \mathrm{~h}$ at room temperature. LST-007 was decanted and the wells were washed 3 times with $200 \mu$ l of PBS containing $0.05 \%$ Tween-20. A secondary antibody consisting of a 1:10,000 dilution of goat anti-human IgG-Fc-HRP conjugate (\#A80-104P; Bethyl) in blocking solution was added for $1 \mathrm{~h}$ at room temperature. Following identical washing as described above, $50 \mu \mathrm{l}$ of TMB solution (\#ES001; Millipore) was added after which the colorimetric reaction was quenched following the addition of $50 \mu \mathrm{l} 10 \% \mathrm{H}_{2} \mathrm{SO}_{4}$. The plates were read in an ELISA reader at OD $450 \mathrm{~nm}$. Blocking buffer with the sole presence of primary or secondary antibodies was used as blank controls.

Binding of LST-007 towards immobilized, whole PA bacteria in ELISA. PA strains were grown in $5 \mathrm{ml} \mathrm{LB}$ medium at $37^{\circ} \mathrm{C}$ in an orbital shaker for $18 \mathrm{~h}$, pelleted and washed twice with PBS. Following resuspension of the bacterial pellet with a small volume of PBS, the volume was adjusted to obtain an OD $600 \mathrm{~nm}$ of 0.2 . To permit bacterial binding, $50 \mu \mathrm{l}$ of poly-L-lysine (PLL) of a $1 \mu \mathrm{g} / \mathrm{ml}$ solution was added to the wells of Maxisorp ELISA plates and incubated for $30 \mathrm{~min}$ at room temperature with orbital shaking. Following removal of PLL, $50 \mu 1$ of the PA suspensions were added to plates and immediately centrifuged at $1,500 \mathrm{rpm}$ for $20 \mathrm{~min}$. Thereafter, supernatants were carefully removed with a multi-channel pipettor and adsorbed PA bacteria were irreversibly fixed to wells by adding $75 \mu 10.2 \%$ formaldehyde for $15 \mathrm{~min}$ at room temperature. Following removal of formaldehyde and brief drying, plates were blocked overnight at $4^{\circ} \mathrm{C}$ with $200 \mu 1$ PBS-10\% FBS. The blocking solution was removed and $50 \mu \mathrm{l}$ of $0.5 \mu \mathrm{g} / \mathrm{ml}$ LST-007, LST-003 or human isotype control $\mathrm{mAb}$ was added for $60 \mathrm{~min}$ at $37^{\circ} \mathrm{C}$. The ELISA was continued in an identical manner with recombinant PA flagellin type $b$ as described above.

Isoelectric focusing (IEF) studies. IEF gels $(5 \% \mathrm{~T} / 3 \% \mathrm{C}$; $0.25 \mathrm{~mm}$ thick) were prepared by polymerization of acrylamide and N,N'-methylenebisacrylamide on GelBond ${ }^{\circledR}$ PAG film $(124 \times 258 \mathrm{~mm})$, followed by washing, drying, and reconstitution with $2 \%$ Pharmalyte $3-10,10 \mathrm{mM}$ glutamic acid, $10 \mathrm{mM}$ lysine, and $32 \%$ (v/v) glycerol. IEF was performed on a horizontal unit, at $15^{\circ} \mathrm{C}$, under a nitrogen atmosphere, $10 \mathrm{~cm}$ between electrodes. Gels were prefocused for $500 \mathrm{~V}-\mathrm{h}$ (500 V, $60 \mathrm{~min})$. Samples were loaded as drops on the gel surface and focused for 9,500 V-h $(500 \mathrm{~V}, 30 \mathrm{~min} ; 1,000 \mathrm{~V}$, $30 \mathrm{~min} ; 2,000 \mathrm{~V}, 30 \mathrm{~min} ; 3,500 \mathrm{~V}, 133 \mathrm{~min}$ ). Gels were fixed in $20 \%(\mathrm{w} / \mathrm{v})$ trichloroacetic acid and stained with Coomassie G-250 using the colloidal methodology (33).

Surface plasmon resonance (SPR) studies. SPR was performed on a Biacore 3000 instrument (Biacore, Uppsala, Sweden). Recombinant PA flagellin type $b$ was diluted in $100 \mathrm{mM}$ sodium acetate ( $\mathrm{pH}$ 4.6) to a final concentration of
$50 \mu \mathrm{g} / \mathrm{ml}$ and immobilized on a CM5 Biacore sensor chip. The antigen was then streamed over the sensor chip for $5 \mathrm{~min}$ at a rate of $10 \mathrm{ml} / \mathrm{min}$. The binding assay was performed by injecting LST-007 at 16 different concentrations ranging from 0.0 to $50 \mathrm{nM}$ at a flow rate of $20 \mathrm{ml} / \mathrm{min}$ at $25^{\circ} \mathrm{C}$. These conditions resulted in a linear relation between the concentration of LST-007 and the maximal (steady-state) response, indicating the pseudo first-order regime in relation to the immobilized ligand. The net signal was obtained by subtracting the blank signal (dextran matrix). The association phase for LST-007 binding was monitored for $4 \mathrm{~min}$, while the dissociation phase for $3 \mathrm{~min}$. Responses were monitored as a function of time by generating the traces (sensorgrams) at $25^{\circ} \mathrm{C}$. Multi-concentration data were globally fitted using the BIAevaluation 3.2 software supplied by Biacore to calculate affinity constant of LST-007.

In vitro PA motility assays. Motility studies were performed as previously described (34). In brief, freshly prepared PA colonies were taken for motility experiments as follows. Soft liquid agar was prepared by autoclaving LB media containing $1 \%$ tryptone, $0.5 \% \mathrm{NaCl}, 0.3 \%$ yeast extract and $0.3 \%$ soft agar. This solution was transferred to a water bath at $40^{\circ} \mathrm{C}$ for $30 \mathrm{~min}$. After $30 \mathrm{~min}$, the solution was aliquoted into working volumes and mAbs or diluted Cmax sera were added at the desired concentrations. In some assays, $10 \mathrm{~cm}$ plates were used whereby $20 \mathrm{ml}$ of the mAb-containing soft agar was poured. In some assays, 24-well plates were used, with $1 \mathrm{ml}$ of the mAb-containing soft agar poured/well. Plates were allowed to solidify for $\sim 30 \mathrm{~min}$ at room temperature within a bacterial culture hood and wells were inoculated with the desired freshly prepared PA strain by picking a colony with a sterile toothpick and carefully stabbing a few $\mathrm{mm}$ into the $\mathrm{mAb}$-impregnated agar. Plates were wrapped in parafilm to prevent dehydration and incubated at $30^{\circ} \mathrm{C}$ for $12-16 \mathrm{~h}$ to maximize flagellin expression. Wells were photographed and the motility was measured as the diameter of the halo phenotype that surrounds the central bacterial growth.

LST-007 pharmacokinetic studies. Female CD-1 mice, age 10-12 weeks (20-25 g) were used. Animal handling was performed according to the National Institute of Health (NIH) and the Association for Assessment and Accreditation of Laboratory Animal Care (AAALAC). During acclimation (5 days) and following LST-007 dosing, mice were housed in a specific pathogen-free environment with 3 mice/cage, in polypropylene cages fitted with solid bottoms and filled with autoclaved sawdust as bedding material. Animals were provided ad libitum with a commercial rodent diet and free access to autoclaved drinking water supplied to each cage. Automatically controlled environment conditions were set to maintain a temperature of $22-25^{\circ} \mathrm{C}$ with a $12 \mathrm{~h}$ light/ $12 \mathrm{~h}$ dark cycle and air changes in the study room.

LST-007 was prepared at a concentration of $1 \mathrm{mg} / \mathrm{ml}$ and injected at $5 \mathrm{ml} / \mathrm{kg}$ to achieve a target dose of $5 \mathrm{mg} / \mathrm{kg}$. A total of 27 mice were taken for simultaneous PK sampling from bleeds and bronchoalveolar lavage (BAL) fluid, with 3 mice/time point sacrificed for both samplings. Sampling time points were $5 \min (\mathrm{Cmax}), 4,8,24,48,96,120,168$ and $240 \mathrm{~h}$. Additionally, 3 mice were sampled at $5 \mathrm{~min}$ following 
the injection of saline at $5 \mathrm{ml} / \mathrm{kg}$. In brief, mice were injected intravenously (i.v.) with LST-007 in the tail vein using a tuberculin syringe and a $30 \mathrm{G}$ needle. At the designated time points, mice were anesthetized using an intraperitoneal (i.p.) injection of $85 \mathrm{mg} / \mathrm{kg}$ xylazine and $5 \mathrm{mg} / \mathrm{kg}$ ketamine and bled, $\sim 500 \mu 1$ from the orbital sinus. The blood was collected into $1.5 \mathrm{ml}$ Eppendorf tubes, centrifuged and the upper sera layer was aliquoted and stored at $-80^{\circ} \mathrm{C}$ until required. While the mice were still under anesthesia, they were placed on their backs and the airway was exposed for collection of BAL fluid by connection of a veinflow to the airway attached to a $26 \mathrm{G}$ needle and $1 \mathrm{ml}$ syringe. A total volume of $700 \mu 1$ of saline was used to wash the lungs with return BAL volumes $\sim 500 \mu \mathrm{l}$. Following centrifugation, the clarified BAL supernatant was removed, aliquoted and stored at $-80^{\circ} \mathrm{C}$ until assay.

For sampling LST-007, an ELISA was adapted whereby dilutions of sera (1:500-1:2,000) or BAL fluid (1:100-1:400) were added to ELISA plates coated with a goat anti-human IgG Fc specific antibody. Following incubation and blocking (see above), wells were incubated with a detecting anti-human $\kappa$-HRP antibody followed by colorimetry with TMB. Plates were read at $450 \mathrm{~nm}$ after quenching with $\mathrm{H}_{2} \mathrm{SO}_{4}$. Since the BAL fluid compartment undergoes 'dilution' via flushing with saline, recovered BAL samples were taken for BUN determinations (AML, Herzliya, Israel), enabling normalization. As an example, BUN levels in BAL were measured at $1.67 \mathrm{mg} / \mathrm{dl}$ as compared to concentrations of $25 \mathrm{mg} / \mathrm{dl}$ in mouse sera. Thus, BAL-derived LST-007 concentrations determined in ELISA were normalized following a 15 -fold multiplication.

Mouse model of pneumonia. Adult C57 mice (25 g; Harlan Nossan, Milan, Italy) were housed in a controlled environment and provided with a standard rodent diet and water. Animal care was in compliance with the Italian regulations for protection of animals used for experimental and other scientific purposes (DM 116192) as well as the EEC regulations (OJ of ECL 358/1 12/18/1986). The mice were housed in cages with filter tops in specific pathogen-free conditions. They were briefly anesthetized with inhaled Sevorane (Abbot Laboratories) in an oxygenated chamber and placed in a supine position with their heads elevated $\sim 30^{\circ}$. Initially, a limited number of mice were dedicated to the animal surgery and intratracheal (i.t.) procedure by validating $100 \%$ animal survival and normal behavior throughout the 7 days post instillation of $50 \mu \mathrm{l}$ lactated Ringer's solution into the left lung. Thereafter, a $\mathrm{LD}_{80}$ pneumonia model was established at 3 days post-infection, elicited by the i.t. administration of the MDR PA strain $\mathrm{Ka} 02$ at $10^{6} \mathrm{cfu}$ in $50 \mu 1$ lactated Ringer's solution. Using this $\mathrm{LD}_{80}$, the biological activity of LST-007 or control treatments (i.v. $6 \mathrm{ml} / \mathrm{kg}$ ) on animal survival were investigated in the following 5 experimental groups: group 1 $(\mathrm{n}=20)$ : i.t. $\mathrm{Ka} 02$ followed by i.v. formulation buffer $(\mathrm{FB})$ at +60 min and $+25 \mathrm{~h}$ after infection; group $2(\mathrm{n}=20)$ : i.t. $\mathrm{Ka} 02$ followed by i.v. LST-007 $(20 \mathrm{mg} / \mathrm{kg})$ at $+60 \mathrm{~min}$ and FB at $+25 \mathrm{~h}$; termed 'LST-007x1'; group $3(\mathrm{n}=20)$ : i.t. Ka02 followed by i.v. LST-007 $(20 \mathrm{mg} / \mathrm{kg})$ at $+60 \mathrm{~min}$ and $+25 \mathrm{~h}(20 \mathrm{mg} / \mathrm{kg})$; termed 'LST-007x2'; group $4(\mathrm{n}=20)$ : i.t. Ka02 followed by freshly prepared, i.p. imipenem $(25 \mathrm{mg} / \mathrm{kg} ; 0.25 \mathrm{ml}$ of a $2.5 \mathrm{mg} / \mathrm{ml}$ solution) given b.i.d. for 3 days post-infection at time points of $+60 \mathrm{~min},+5,+24,+29,+48$ and $+53 \mathrm{~h}$;

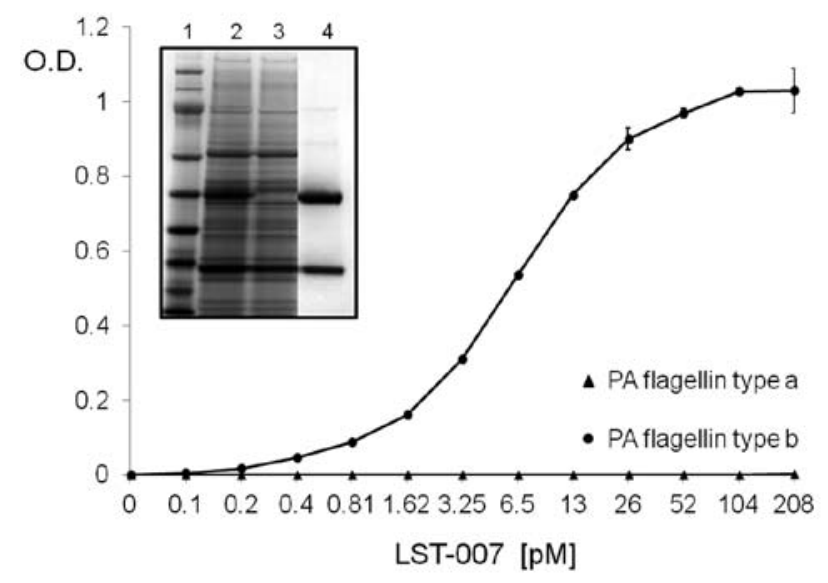

$(-) \begin{array}{llllll}9.3 & 8.5 & 7.4 & 6.6 & 5.2 & 3.5(+)\end{array}$

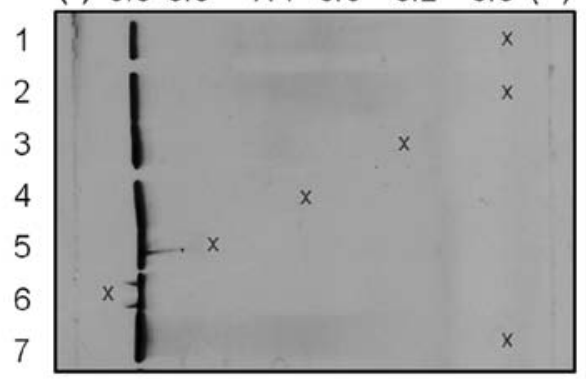

Figure 1. In vitro properties of recombinant LST-007 expressed and purified from CHO cells. Upper panel depicts purification profile of LST-007 as shown by Coomassie gel staining of clarified $\mathrm{CHO}$ cell supernatant (lane 2), wash fraction (lane 3) and eluted LST-007 product (lane 4), under reducing conditions. Lane 1 is a Mw (kDa) marker of sizes 191, 97, 64, 51, 39, 28, 19 and 14. ELISA curve demonstrates concentration-dependent binding of LST-007 from at least 3 independent experiments towards E. coli-expressed, purified PA flagellin type $b$ (circles) with no reactivity towards flagellin type a (triangles). Lower panel demonstrate IEF properties of LST-007. Samples of $2 \mathrm{mg} / \mathrm{ml}$ LST-007 were loaded as $5 \mu 1$ drops to the gel surface at designated positions (denoted by x) at $1 \mathrm{~cm}$ (lane 2), $3 \mathrm{~cm}$ (lane 3 ), $5 \mathrm{~cm}$ (lane 4 ), $7 \mathrm{~cm}$ (lane 5) and $9 \mathrm{~cm}$ (lane 6) from the anode. Additionally, LST-007 was loaded at $1 \mathrm{~cm}$ from the anode at a concentration of $1 \mathrm{mg} / \mathrm{ml}$ (lane 1) and $5 \mathrm{mg} / \mathrm{ml}$ (lane 7). The results show that LST-007 is a very basic protein of pI 9.3.

group $5(\mathrm{n}=15)$ : i.t. $\mathrm{Ka} 02$ followed by i.v. human isotype $\mathrm{mAb}$ $(20 \mathrm{mg} / \mathrm{kg})$ at $+60 \mathrm{~min}$ and $+25 \mathrm{~h}(20 \mathrm{mg} / \mathrm{kg})$. For groups 2,3 and $5, \mathrm{mAb}$ stock concentrations of $3.33 \mathrm{mg} / \mathrm{ml}$ were provided from which $0.15 \mathrm{ml}$ were injected per mouse (i.e. $6 \mathrm{ml} / \mathrm{kg}$ ).

Statistics were run in Stata version 7 (Stata Corp., College Station, TX, USA). The differences between the antibody or imipenem treated groups vs. FB-treated groups were analyzed. Dichotomous outcomes were compared using Fisher's exact test and continuous variables by the Student's t-test. All statistical tests were two-tailed. Differences were considered to be statistically significant with a P-value $\leq 0.05$.

\section{Results}

In vitro characterization of mammalian-expressed LST-007. Recombinant LST-007 was expressed in CHO cells and purified to homogeneity. The upper panel of Fig. 1 shows the Coomassie gel staining of an SDS-PAGE loaded with clarified media containing secreted LST-007 (lane 2), a washed fraction (lane 3) and $4 \mu \mathrm{g}$ of the final eluted LST-007 product (lane 4) depicting the heavy and light chains. Lane 1 is a 


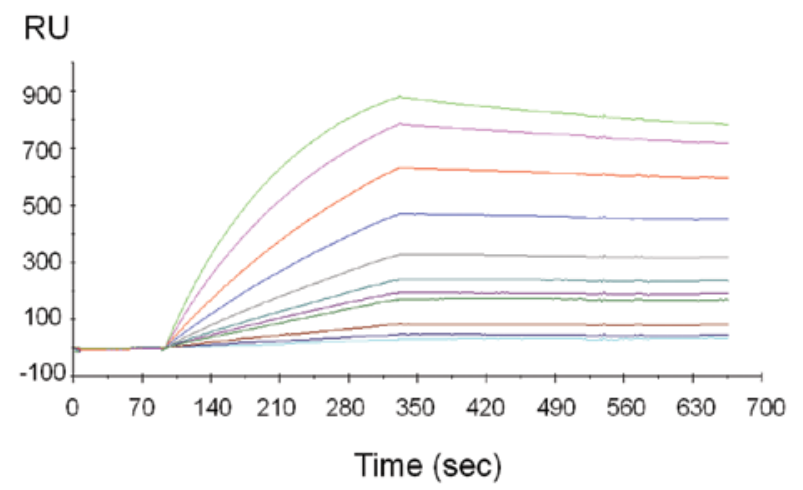

Figure 2. Concentration-dependent, sensorgram traces of LST-007 binding towards purified, E.coli-expressed PA flagellin type b by surface plasmon resonance (SPR) using Biacore 3000. Binding of purified LST-007 towards its purified antigen on coated CM5 biosensor chips. Sensorgram traces depict binding of LST-007 at $0.5,0.75,1,2.5,3,4,5,7.5,10,15$ and $20 \mathrm{nM}$ with proportional increases in resonance units (RU). The affinity of LST-007 affinity towards recombinant PA flagellin type b was calculated at $7.4 \times 10^{-10} \mathrm{M}$.

molecular weight (Mw) marker of bands 14, 19, 28, 39, 51, 64, 97 and $191 \mathrm{kDa}$. LST-007, prepared as a sterile-filtered solution at $13.15 \mathrm{mg} / \mathrm{ml}$, was verified to be free of bacterial burden with LPS $<0.04 \mathrm{EU} / \mathrm{mg}$. In ELISA studies employing recombinantly expressed flagellin type $b$ as the immobilized antigen, LST-007 demonstrated a concentration-dependent increase in OD which peaked at $208 \mathrm{pM}(=31.25 \mathrm{ng} / \mathrm{ml}$ LST-007). No binding was observed whatsoever with PA flagellin type a (Fig. 1). In IEF studies, when loaded at various locations on the IEF gel at different amounts, LST-007 was demonstrated to be a very basic protein with a $\mathrm{pI}$ of 9.3 (Fig. 1, lower panel). When added maximally at $25 \mu \mathrm{g}$ (lane 9), two very light additional bands were observed next to the main band at $\mathrm{pH} 9.2$. While it is possible that the heavy $\mathrm{pH} 9.3$ band consists of several unresolved bands, for the most part, the isoelectric focusing data at $\mathrm{pH} 9.2$ demonstrates a high level of LST-007 electrostatic homogeneity. The basic pI of LST-007 is similar to the pIs of a number of marketed mAbs from theoretical and experimental measurements (35).

The specificity of the above ELISA data was re-enforced in surface-plasmon resonance (SPR) studies. Here, LST-007 (0.5-20 nM) was streamed over immobilized PA flagellin type $b$ bound to a sensor chip and concentration-dependent increases in the signal were observed as demonstrated in the sensorgrams (Fig. 2). Analysis of these data confirmed the high affinity of LST-007 towards recombinant PA flagellin type $b$ which was calculated at $0.74 \mathrm{nM}$.

To further scrutinize the specificity of LST-007 binding and assess its capability to bind the 'naturally-occurring' flagellin type b, ELISA studies were established whereby the PA target was in the form of immobilized whole PA bacteria tethered to ELISA plates via poly-L-lysine (PLL). In these binding studies (Fig. 3), PA strains 27853 (type a flagellin), Pa01 (type b flagellin) and 2 additional flagellin type b containing PA strains Ka02 and 25619 (as determined by PCR analysis, data not shown) were immobilized on plates. Additionally, a non-motile PA strain (9721) and thus potentially devoid of a flagellum was also incorporated in the

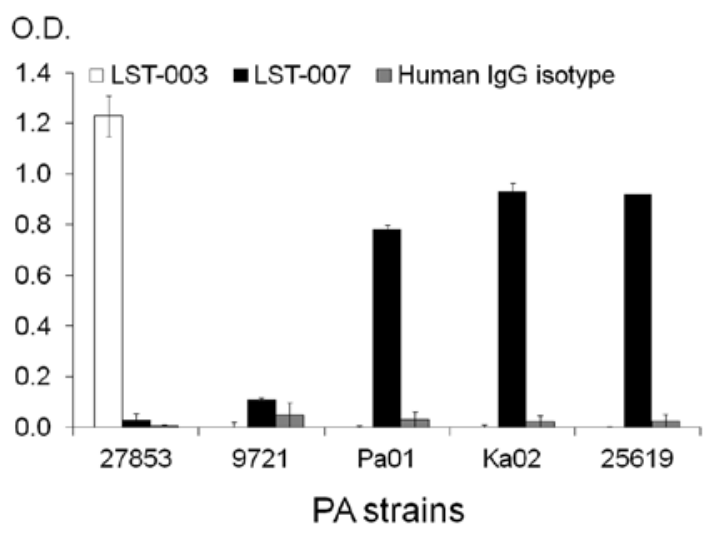

Figure 3. LST-007 demonstrates specific binding towards immobilized whole PA bacteria harboring flagellin type b, but not type a, in fixed ELISA. Binding of LST-007 $(0.5 \mu \mathrm{g} / \mathrm{ml})$ was investigated towards formaldehydefixed, intact PA bacterial strains known to express type a (Pa27853) or type b (Pa01, Ka02, 25619) flagellin. Additionally, a non-motile PA strain (9721) was used as a control. LST-007 demonstrated specific binding towards all PA type b flagellin strains with no binding towards Pa27853 or 9721. A proprietary chimeric mAb termed LST-003, which solely binds PA flagellin type a, served as a control in the ELISA screen. No binding to any of the PA strains was observed with a human isotype control $\mathrm{mAb}$ at $0.5 \mu \mathrm{g} / \mathrm{ml}$.

screen as a negative control. LST-007 $(0.5 \mu \mathrm{g} / \mathrm{ml})$ specifically bound strains PAO1, Ka02 and 25619 but was devoid of reactivity towards PA strains 27853 and 9721 . Conversely, LST-003 solely reacted with strain 27853 . To confirm the specificity of the ELISA, $0.5 \mu \mathrm{g} / \mathrm{ml}$ of a human isotype control mAb failed to react with any of the PA strains (Fig. 3). These data therefore confirm that LST-007 is a highly specific $\mathrm{mAb}$ and capable of binding flagellin type $\mathrm{b}$ in its natural form as part of intact PA bacteria.

In vitro biological activity of LST-007 on perturbation of PA motility in soft agar assays. Based on the proven, high specific reactivity of LST-007 towards PA flagellin type $b$ and the quality of the mAb preparation, bioactivity studies were undertaken by assessing the capability of LST-007 to perturb PA motility in soft-agar impregnated with the mAb. In such studies, LST-007 from disparate sources was employed as follows: i) using Cmax sera from our PK studies whereby sera were diluted from 1:100-1:3,200, and ii) using exogenously added LST-007 from our purified mAb preparation at identical concentrations to the measured serum titer based on the LST-007 Cmax of $804.86 \mathrm{nM}(120.67 \mu \mathrm{g} / \mathrm{ml})$ (Table I). Concentration-dependent inhibition profiles on PA motility was observed with either the ex vivo (Fig. 4A) or exogenous source (Fig. 4B) of LST-007 with complete suppression of PA motility observed at $6.6 \mathrm{nM}(\sim 1 \mu \mathrm{g} / \mathrm{ml}) \mathrm{mAb}$.

PK profile of LST-007 in blood and broncholveolar lavage (BAL) fluid from naïve mice. The PK profile of LST-007 in blood and BAL fluid was investigated following a single i.v. injection of the $\mathrm{mAb}$ at a dose of $5 \mathrm{mg} / \mathrm{kg}$ in naïve mice. This dose was chosen based on a PK study performed with an anti-RAGE mAb (36) and deemed appropriate by us in terms of our ELISA detection capabilities based on the theoretical concentrations of LST-007 expected in different compartments. In sera, LST-007's Cmax (at 5 min) was $804.86 \mathrm{nM}$ 


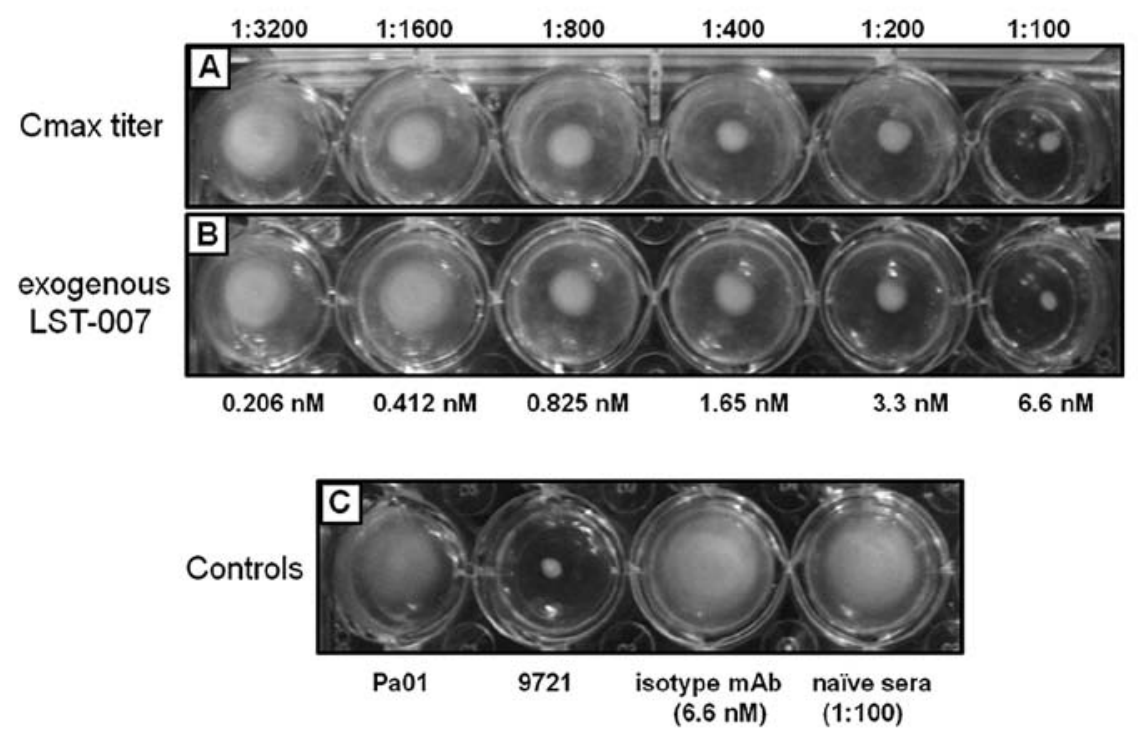

Figure 4. Photomicrographs showing the de novo inhibitory effect of LST-007 on PA motility in soft agar assays. Single Pa01 colonies were stabbed into soft agar impregnated with different dilutions of (A) PK-derived pooled Cmax sera (1:3200-1:100) or (B) equivalent calculated amounts of exogenously added LST-007 (0.206-6.6 nM). LST-007 from both sources caused identical, dose-dependent inhibition of bacterial motilities as indicated by the reduced halo effect encircling the origin of bacterial stabbing and growth. (C) No effect on bacterial motility was observed at a 1:100 dilution of naïve mice sera nor 6.6 $\mathrm{nM}$ of an irrelevant human isotype mAb. The phenotype of a non-motile bacterial strain 9721 is depicted as well as Pa01 alone.

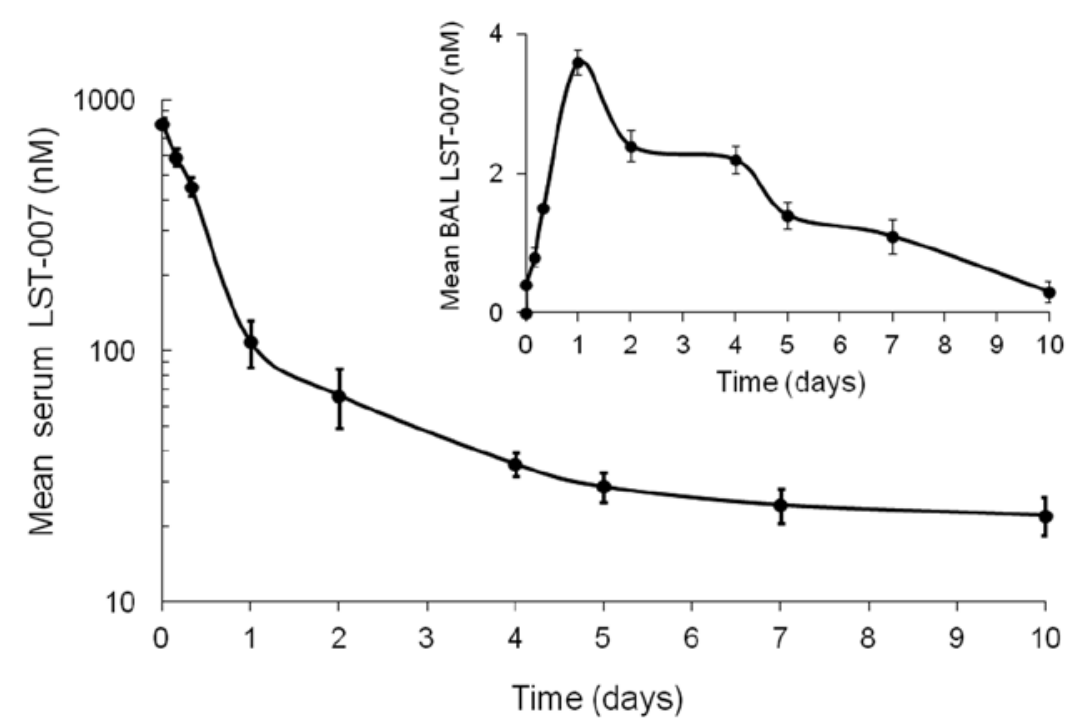

Figure 5. PK profile of LST-007 in sera and BAL fluid from naïve mice. Naïve CD mice were injected i.v. with LST-007 (5 mg/kg) and sacrificed at the various time points shown for simultaneous blood and BAL fluid LST-007 determinations using a highly specific human ELISA. In blood, LST-007 demonstrated a rapid elimination phase during the first $24 \mathrm{~h}$ with a much slower phase of elimination up to Day 10. LST-007's half-life in blood was calculated to be $83.5 \mathrm{~h}$. In contrast to blood, BAL fluid LST-007 concentrations (inset) peaked at $24 \mathrm{~h}$ and slowly decreased over the ensuing 9 days.

(120.67 $\mu \mathrm{g} / \mathrm{ml})$ followed by a rapid phase of elimination to a concentration of $111.18 \mathrm{nM}(16.67 \mu \mathrm{g} / \mathrm{ml})$ at $24 \mathrm{~h}$ (Fig. 5). A much slower rate of elimination occurred over the next 9 days since the LST-007 concentration was measured at $26.6 \mathrm{nM}(4 \mu \mathrm{g} / \mathrm{ml})$ at Day 10 (Fig. 5). LST-007's half-life was calculated to be $83.58 \mathrm{~h}$ (Table I).

In marked contrast to the blood compartment kinetics, LST-007 exhibited a different PK profile in BAL fluid since $\mathrm{mAb}$ concentrations increased over the first $24 \mathrm{~h}$, peaking to $3.6 \mathrm{nM}(0.54 \mu \mathrm{g} / \mathrm{ml})$ at the $24 \mathrm{~h}$ time point and decreased slowly over the next 9 days (Fig. 5). LST-007 was still detectable in BAL fluid at Day 10 at a concentration of $0.3 \mathrm{nM}$ $(0.045 \mu \mathrm{g} / \mathrm{ml})$.

Effect of LST-007 on animal survival in a lethal MDR $P A$-induced model of pneumonia. The biological effect of LST-007 on animal survival was investigated in a lethal mouse model of pneumonia driven by an MDR PA strain (Ka02). Initial calibration studies demonstrated that i.t. instillation of $10^{6}$ cfu $\mathrm{KaO} 2$ elicited an $\mathrm{LD}_{80}$ at 3 days (data not shown). 


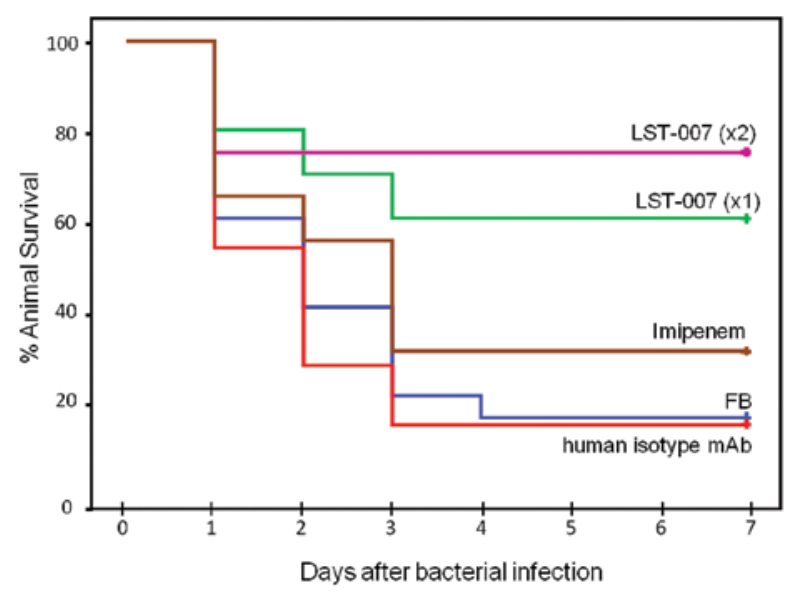

Figure 6. LST-007 markedly improves the survival of mice in a lethal model of MDR PA-induced pneumonia. Mice were infected intra-tracheally with $10^{6} \mathrm{cfu} \mathrm{Ka02}\left(\mathrm{LD}_{80}\right)$ and treated with LST-007 $(20 \mathrm{mg} / \mathrm{kg})$ at $60 \mathrm{~min}$ postinfection (LST-007x1) in one group $(\mathrm{n}=20)$ or with an additional dose $+25 \mathrm{~h}$ (LST-007x2) in a second group $(\mathrm{n}=20)$. Other experimental groups included LST-007 formulation buffer ( $\mathrm{FB} ; \mathrm{n}=20$ ), a human isotype control $\mathrm{mAb}$ ( $20 \mathrm{mg} / \mathrm{kg} ; \mathrm{n}=15$ ) or imipenem ( $25 \mathrm{mg} / \mathrm{kg}$, bid for 3 days; $\mathrm{n}=20$ ). Survival was assessed for up to 7 days following infection. By Fisher's exact test, $\mathrm{P}=0.0002$ for LST-007 (x1) and P<0.0001 for LST-007 (x2) as compared to the FB group. Imipenem treatment was not significant $(\mathrm{P}=0.1553)$ vs. the $\mathrm{FB}$ group.

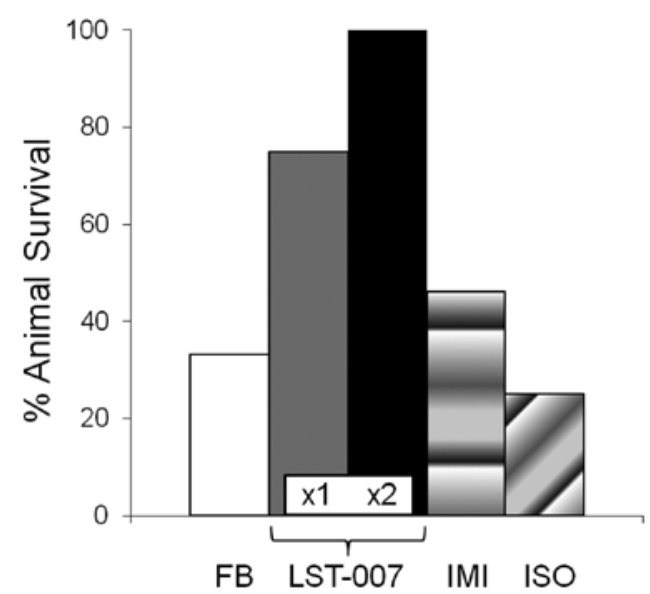

Figure 7. LST-007 (x2) affords complete animal survival between Days 1 and 3 post-infection. The effect of a second i.v. administration of LST-007 $(20 \mathrm{mg} / \mathrm{kg}$ ) given $24 \mathrm{~h}$ after infection on survival was investigated. This second dose of LST-007 completely prevented the further decrease in mortality observed with LST-001 (x1) dosing paradigm between 24 and $72 \mathrm{~h}$ after infection, allowing 100\% survival of mice. LST-007 (x2) effect was specific since two i.v. injections of a human isotype $\mathrm{mAb}$ (ISO) at identical dosing to LST-007 failed to attenuate mortality changes during the same period. FB, formulation buffer; IMI, imipenem.

This mortality profile was used by us in subsequent feasibility studies with LST-007. Following lung infection, mice treated with i.v. FB demonstrated typical lethality curves with 60, 40 and 20\% survival at Days 1, 2 and 3, respectively (Fig. 6). In marked contrast, infected animals treated with LST-007x1 or LST-007x2 demonstrated marked improvements in survival at 1-3 days post-infection with 60 and $75 \%$ survivors at Day 3 respectively which remained unchanged until Day 7 (Fig. 6). The specificity of this effect was confirmed since an irrelevant human isotype control $\mathrm{mAb}$ administered at $20 \mathrm{mg} / \mathrm{kg}$ twice
Table I. Pharmacokinetic analysis of LST-007 in naïve mice blood following the i.v. injection of a single dose of $5 \mathrm{mg} / \mathrm{kg}$.

LST-007 PK analysis

\begin{tabular}{lccc}
\hline AUC & $(0$ to 240$)$ & $\mathrm{mvc} / \mathrm{ml} * \mathrm{~h}$ & 2463.34 \\
& (0 to infinity) & $\mathrm{mcg} / \mathrm{ml} * \mathrm{~h}$ & 2772.88 \\
Tmax & $(0$ to 240$)$ & $\mathrm{h}$ & 0.083 \\
Cmax & $(0$ to 240$)$ & $\mathrm{mcg} / \mathrm{ml}$ & 120.67 \\
Cmin & $(0$ to 240$)$ & $\mathrm{mcg} / \mathrm{ml}$ & 2 \\
Cavg & $(0$ to 240$)$ & $\mathrm{mcg} / \mathrm{ml}$ & 10.26 \\
& $\mathrm{Ke}$ & $1 / \mathrm{h}$ & 0.009565 \\
& $\mathrm{~T} 1 / 2$ & $\mathrm{~h}$ & 83.58 \\
\hline
\end{tabular}

at +1 and $+25 \mathrm{~h}$, failed to curtail mortality which essentially superimposed on the mortality profile observed with the FB treatment group (Fig. 6). Importantly, imipenem treatment at doses and a route of administration known to be protective against the laboratory strain PAO1 $(30,31)$ was ineffective in preventing $\mathrm{KaO} 2$-induced mortality with survival of only $30 \%$ at Day 3 (Fig. 6). The importance and significance of a second dosing of LST-007 is underscored in Fig. 7 since the $\mathrm{mAb}$ completely abrogated mortality changes noted with LST-007x 1 at Day 2 (2/16 deaths) and Day 3 (2/14 deaths).

\section{Discussion}

A call has been heeded by the Infectious Diseases Society of America (IDSA) and the European Medicines Agency (EMA) for the urgent development of novel antibacterial strategies targeting in particular, the ESKAPE group of pathogens (1,37). In the case of Pseudomonas aeruginosa (PA), a major dearth exists due to the lack of effective antibacterials targeting MDR PA strains. Currently, most of the anti-PA drugs in late stage pre-clinical testing or early clinical evaluation are modifications of existing drugs, newer combinations of existing drugs or even newer routes of administration of old drugs (38-40). Clearly, innovative remedies are urgently required to combat MDR PA which is a major nosocomial threat, especially in immunocompromised patients.

It is axiomatic that mAbs are proven therapeutics for cancer and immunologic disorders (41), with increasing recognition that $\mathrm{mAbs}$ may serve as bona fide drugs for a variety of infectious diseases $(42,43)$ including PA $(19,20)$ by targeting surface-expressed virulence factors. We developed and expressed in CHO cells, a fully human mAb (LST-007) against the flagella of PA by targeting flagellin type $b$, one of the two major protein forms of this appendage. LST-007 was purified to homogeneity from $\mathrm{CHO}$ cells, reacted specifically with $E$. coli-expressed flagellin type b and exhibited a high affinity of $7.4 \times 10^{-10} \mathrm{M}$ in SPR assays. These binding profiles were supported by the capability of LST-007 to selectively bind its native target in whole PA bacteria. Since flagellin type $b$ is glycosylated in PA (44) and may have an impact on mAb binding, integrity of LST-007's recognition towards PA in the fixed ELISA format served as an impetus for bioactivity assays. 
Previously, anti-PA flagellin mAbs were reported to inhibit PA motility in vitro which presumably laid the basis for their beneficial effects in models of PA infection (27-29). Similarly, LST-007 suppressed PA motility at concentrations close to its $K_{D}$ of the mAbs. Furthermore, we also demonstrated that hyperimmune sera derived from PK studies with LST-007 was similarly bioactive to exogenously added mAb (Fig. 4). This ex vivo finding was important for 2 reasons: i) it demonstrated that LST-007 retains bioactivity in vivo indicating its likely stability within blood, and ii) it provided us with valuable information regarding LST-007's target concentration that should allow effective inhibition of PA motility and positive proof-of-concept in our pneumonia model. Indeed, a 1:100 dilution of Cmax sera containing $\sim 1 \mu \mathrm{g} / \mathrm{ml}$ LST-007 (6.6 nM LST-007) can be regarded as the minimal inhibitory concentration (MIC) that caused complete inhibition of PA motility by comparing its phenotype in soft agar with 9721 , the non-motile aflagellated PA strain (Fig. 4).

The PK profile of LST-007 in the blood from naïve mice demonstrated a half-life of $\sim 84 \mathrm{~h}$, reaching a concentration $\sim 15 \%$ of the Cmax at $24 \mathrm{~h}$ during a rapid elimination phase. At such a concentration $(16 \mu \mathrm{g} / \mathrm{ml}), \sim 10 \mathrm{X}$ MIC on motility, LST-007 may be expected to be bioactive in a bloodstream PA infection. Nevertheless, LST-007 bioactivity in a pneumonia model is governed by its traversing into the alveolar space from blood. LST-007 concentrations from the same naïve mice were measured in BAL fluid, peaked at $24 \mathrm{~h}$ $(\mathrm{Cmax}, 0.5 \mu \mathrm{g} / \mathrm{ml})$ and gradually decreased until Day 10 (Fig. 5). Based on an 0.5 MIC of LST-007 in BAL fluid at $24 \mathrm{~h}$ after dosing with $5 \mathrm{mg} / \mathrm{kg}$, we rationalized that a higher and potential additional dose of LST-007 may be required to allow appropriate pharmacodynamic 'coverage' and a desired biological effect in the pneumonia model. When administered a single dose of $20 \mathrm{mg} / \mathrm{kg}$, LST-007 improved survival at each day during the first 3 days during which significant mortality occurred in both control groups. Importantly, a second dose of LST-007, $25 \mathrm{~h}$ after infection, completely attenuated mortality changes that occurred at Days 2 and 3. These latter findings would indicate that LST-007 reached the alveolar space at concentrations exceeding $1 \mathrm{X}$ MIC which are sustained during the 3 days mortality window. Clinical implications from these findings are 2-fold: i) constant infusion of LST-007 during PA colonization and infection may be the appropriate mode of administration, and ii) LST-007 could be given prophylactically or at least during PA colonization since it traverses into the alveolar space of intact lung. This latter property is presumably due to LST-007 being an IgG1 isotype since the accumulation of the IgM mAb panobacumab in BAL fluid was critically dependent upon the consequences of PA infection (i.e. lung microvascular permeability defects) (17).

A limitation in the present study is that bacterial burden in lungs was not measured. However, since the infecting strain was instilled directly into the lung and there is no evidence that anti-PA mAbs can exert bactericidal effects, reduction in lung bacterial burden are unlikely yet still needs to be verified. Nevertheless, LST-007-mediated suppression of infection throughout the lung or to other organs may occur as reported in the suppression of bacterial dissemination to the spleen in a burn model of PA infection with an anti-type a flagellin $\mathrm{mAb}$ (31). Taken together, our data indicated that the beneficial effect of LST-007 on survival may be due to factors that not only target PA motility and accessibility of the bacteria to the TLR5, but also reduced invasiveness into alveolar epithelial cells as well as potential toning down of local inflammatory events driven by monomeric flagellin $(23,45)$. Studies utilizing human alveolar A549 epithelial cells, recombinant flagellins, appropriate infecting PA strains as well as the nonmotile 9721 strain, could help to clearly scrutinize the roles of the flagellum in such processes.

Alanine-scanning mutagenesis has shown that the structural requirements for flagellar motility are much more rigid than the permissive nature of TLR5 recognition of flagellin. (23). Interestingly, mouse mAbs raised against PA flagella (27) which impeded motility were shown to bind PA flagellins downstream from amino acid 161 by SDS-PAGE (31). It may be possible that LST-007 similarly targets PA flagellin type $b$, especially since mutations of a number of conserved $\mathrm{C}^{\prime}$ terminal amino acid residues in flagellin's D1 domain resulted in complete abrogation of bacterial motility (23). Appropriate epitope mapping using techniques such as a hybrid $\beta$-lactamase display as recently demonstrated for an anti-CD22 immunotoxin CAT-8015 (46), could be very useful to localize LST-007's site of binding within PA flagellin type $b$.

From a historical perspective, it is perplexing as to why therapeutic antibodies targeting PA flagellin failed to come to fruition yet reached significant stature in the early 1990's. Cessation of developmental efforts were probably swayed by the disappointing clinical studies with anti-sepsis agents including mAbs $(47,48)$. Additionally, the need to develop two separate mAbs targeting flagellin type $a$ and $b$ for complete anti-PA coverage may have been an issue, especially during a period where many of the methodological aspects concerning the development of therapeutic mAbs were still in their infancy. Today, such issues can be overcome, as exemplified by the prior screening of infected PA patients to ensure compatibility for immunotherapy (19), streamlined $\mathrm{mAb}$ manufacturing approaches (14) or even potential design of bispecific mAbs (49). Flagellin represents a viable target for therapeutic intervention which is further fueled by two additional findings. In translational studies, whereby polymorphisms in the TLR5 and subsequent flagellin hyporesponsiveness are associated with improved health indicators in Crohn's disease (50), CF (51) and systemic lupus erythematosus (52). Secondly, the highly encouraging clinical data in CF patients using oral chicken IgY (53) whereby PA flagellin was proven to be the major immunoreactive antigen bound by this polyclonal therapeutic from proteomic studies (54).

In summary, LST-007 represents a bona fide fully human IgG1 mAb targeting PA flagellin type $b$ which was shown to afford significant improvement in survival against an MDR PA-induced pneumonia, superceding standard care of the treatment. In an era of limited therapeutics targeting MDR PA, neutralizing anti-PA mAbs may not only provide a new monotherapeutic strategy but could additionally synergize with existing antibiotics. Such a desired effect could shepherd these precious drugs from resistance mechanisms. Our current data provide us with significant momentum in continuing the development of LST-007 with the intent of evaluating its clinical efficacy. Such patients could include those within the 
critical care setting (e.g. ventilator-associated pneumonia) or $\mathrm{CF}$ patients. Alternate indications, especially those where PA may be the sole infectious culprit (e.g. urinary tract infections, necrotizing malignant external otitis), could also represent highly feasible patient populations to demonstrate clinical proof-of-concept.

\section{Acknowledgements}

We are grateful to Dr Aharon Rabinkov (Department of Biological Services, Weizmann Institute of Science, Rehovot, Israel) for providing technical support in the Biacore experiments and Mr. Yariv Shoshany for performing the PK analysis. Lostam BioPharmaceuticals Ltd. is truly indebted to the Office of the Chief Scientist (Incubator Program) of the Ministry of Industry, Trade and Labor, Israel for funding all aspects of this project with infrastructure company support under the auspices of the New Generation Technology (NGT) Incubator, Nazareth, Israel.

\section{References}

1. Boucher HW, Talbot GH, Bradley JS, et al: Bad bugs, no drugs: no ESKAPE! An update from the Infectious Diseases Society of America. Clin Infect Dis 48: 1-12, 2009.

2. ECDC/EMEA Joint Technical Report. The Bacterial Challenge: Time to React. A call to narrow the gap between multidrug-resistant bacteria in the EU and the development of new antibacterial agents. pp1-42, 2009. EMEA doc. ref. EMEA/576176/2009 Stockholm, September 2009. doi 10.2900/2518.

3. Driscoll JA, Brody SL and Kollef MH: The epidemiology, pathogenesis and treatment of Pseudomonas aeruginosa infections. Drugs 67: 351-368, 2007.

4. Levin AS and Oliveira MS: The challenge of multidrug resistance: the treatment of gram-negative rod infections. Shock 30: 30-33, 2008.

5. Fujitani S, Sun HY, Yu VL and Weingarten JA: Pneumonia due to Pseudomonas aeruginosa: part I: epidemiology, clinical diagnosis, and source. Chest 139: 909-919, 2011.

6. Pallett $\mathrm{A}$ and Hand K: Complicated urinary tract infections: practical solutions for the treatment of multiresistant Gramnegative bacteria. J Antimicrob Chemother 65: S25-S33, 2010.

7. Flume PA, O'Sullivan BP, Robinson KA, et al: Cystic fibrosis pulmonary guidelines: chronic medications for maintenance of lung health. Am J Respir Crit Care Med 176: 957-969, 2007.

8. Cohen-Cymberknoh M, Shoseyov D and Kerem E: Managing cystic fibrosis: strategies that increase life expectancy and improve quality of life. Am J Respir Crit Care Med 183: 1463-1471, 2011.

9. Hoiby N: Recent advances in the treatment of Pseudomonas aeruginosa infections in cystic fibrosis. BMC Med 9: 32, 2011.

10. Stover CK, Pham XQ, Erwin AL, et al: Complete genome sequence of Pseudomonas aeruginosa PAO1, an opportunistic pathogen. Nature 406: 959-964, 2000.

11. Dean CR, Visalli MA, Projan SJ, Sum PE and Bradford PA: Efflux-mediated resistance to tigecycline (GAR-936) in Pseudomonas aeruginosa PAO1. Antimicrob Agents Chemother 47: 972-978, 2003.

12. Chastre J and Fagon JY: Ventilator-associated pneumonia. Am J Resp Crit Care Med 165: 867-903, 2002.

13. Cegelski L, Marshall GR, Eldridge GR and Hultgren SJ: The biology and future prospects of antivirulence therapies. Nature Rev Microbiol 6: 17-27, 2008.

14. Chartrain M and Chu L: Development and production of commercial therapeutic monoclonal antibodies in mammalian cell expression systems: an overview of the current upstream methodologies. Curr Pharm Biotechnol 9: 447-467 2008.

15. De Jesus $M$ and Wurm FM: Manufacturing recombinant proteins in kg-ton quantities using animal cells in bioreactors. Eur J Pharm Biopharm 78: 184-188, 2011.
16. Baer M, Sawa T, Flynn P, et al: An engineered human antibody fab fragment specific for Pseudomonas aeruginosa PcrV antigen has potent antibacterial activity. Infect Immun 77: 1083-1090, 2009.

17. Secher T, Fauconnier L, Szade A, et al: Anti-Pseudomonas aeruginosa serotype O11 LPS immunoglobulin M monoclonal antibody panobacumab (KBPA101) confers protection in a murine model of acute lung infection. J Antimicrob Chemother 66: 1100-1109, 2011.

18. Horn MP, Zuercher AW, Imboden MA, et al: Preclinical in vitro and in vivo characterization of the fully human monoclonal IgM antibody KBPA101 specific for Pseudomonas aeruginosa serotype IATS-O11. Antimicrob Agents Chemother 54: 2338-2344, 2010.

19. Lu Q, Rouby JJ, Laterre PF, et al: Pharmacokinetics and safety of panobacumab: specific adjunctive immunotherapy in critical patients with nosocomial Pseudomonas aeruginosa $\mathrm{O} 11$ pneumonia. J Antimicrob Chemother 66: 1110-1116, 2011.

20. Milla CE, Accurso FJ, Chmiel J, et al: Modulating Pseudomonas aeruginosa chronic inflammation with the anti-PcrV antibody KB001: Results of a pilot clinical and pharmacodynamic study in subjects with cystic fibrosis. Am J Respir Crit Care Med 181: A1845, 2010.

21. Samatey FA, Imada K, Nagashima $S$, et al: Structure of the bacterial flagellar protofilament and implications for a switch for supercoiling. Nature 410: 331-337, 2001.

22. Yonekura K, Maki-Yonekura S and Namba K: Complete atomic model of the bacterial flagellar filament by electron cryomicroscopy. Nature 424: 643-650, 2003.

23. Smith KD, Andersen-Nissen E, Hayashi F, et al: Toll-like receptor 5 recognizes a conserved site on flagellin required for protofilament formation and bacterial motility. Nat Immunol 4: 1247-1253, 2003.

24. Ansorg RA, Knoche ME, Spies AF and Kraus CJ: Differentiation of the major flagellar antigens of Pseudomonas aeruginosa by the slide coagglutination technique. J Clin Microbiol 20: 84-88, 1984.

25. Montie TJ and Anderson TR: Enzyme-linked immunosorbent assay for detection of Pseudomonas aeruginosa $\mathrm{H}$ (flagellar) antigen. Eur J Clin Microbiol Infect Dis 7: 256-260, 1988.

26. Morgan JA, Bellingham NF, Winstanley C, Ousley MA, Hart CA and Saunders JR: Comparison of flagellin genes from clinical and environmental Pseudomonas aeruginosa isolates. Appl Environ Microbiol 65: 1175-1179, 1999.

27. Rosok MJ, Stebbins MR, Connelly K, Lostrom ME and Siadak AW: Generation and characterization of murine anti flagellum monoclonal antibodies that are protective against lethal challenge with Pseudomonas aeruginosa. Infect Immun 58: 3819-3828, 1990.

28. Ochi $\mathrm{H}$, Ohtsuka $\mathrm{H}$, Yokota $\mathrm{S}$, et al: Inhibitory activity on bacterial motility and in vivo protective activity of human monoclonal antibodies against flagella of Pseudomonas aeruginosa. Infect Immun 59: 550-554, 1991.

29. Landsperger WJ, Kelly-Wintenberg KD, Montie TC, et al: Inhibition of bacterial motility with human antiflagellar monoclonal antibodies attenuates Pseudomonas aeruginosa-induced pneumonia in the immunocompetent rat. Infect Immun 62: 4825-4830, 1994.

30. Neville LF, Barnea Y, Hammer-Munz O, et al: Antibodies raised against N'-terminal Pseudomonas aeruginosa flagellin prevent mortality in lethal murine models of infection. Int J Mol Med 16: $165-171,2005$.

31. Barnea Y, Carmeli Y, Neville LF, et al: Therapy with antiflagellin A monoclonal antibody limits Pseudomonas aeruginosa invasiveness in a mouse burn wound sepsis model. Burns 35 : 390-396, 2009.

32. Eren R, Landstein D, Terkieltaub D, et al: Preclinical evaluation of two neutralizing human monoclonal antibodies against hepatitis $\mathrm{C}$ virus (HCV): a potential treatment to prevent $\mathrm{HCV}$ reinfection in liver transplant patients. J Virol 80: 2654-2664, 2006.

33. Neuhoff V, Arold N, Taube D and Ehrhardt W: Improved staining of proteins in polyacrylamide gels including isoelectric focusing gels with clear background at nanogram sensitivity using Coomassie Brilliant Blue G-250 and R-250. Electrophoresis 9: 255-262, 1988.

34. Rashid MH and Kornberg A: Inorganic polyphosphate is needed for swimming, swarming, and twitching motilities of Pseudomonas aeruginosa. Proc Natl Acad Sci USA 97: 4885-4890, 2000.

35. Boswell CA, Tesar DB, Mukhyala K, Theil FP, Fielder PJ and Khawli LA: Effects of charge on antibody tissue distribution and pharmacokinetics. Bioconjug Chem 21: 2153-2163, 2010. 
36. Vugmeyster Y, DeFranco D, Pittman DD and Xu X: Pharmacokinetics and lung distribution of a humanized anti-RAGE antibody in wild-type and $\mathrm{RAGE}^{-/-}$mice. MAbs 2: $571-575,2010$

37. Freire-Moran L, Aronsson B, Manz C, et al: Critical shortage of new antibiotics in development against multidrug-resistant bacteria - time to react is now. Drug Resist Updat 14: 118-124, 2011.

38. Page MG and Heim J: Prospects for the next anti-Pseudomonas drug. Curr Opin Pharmacol 9: 558-565, 2009.

39. Talbot GH: What is in the pipeline for Gram-negative pathogens? Expert Rev Anti Infect Ther 6: 39-49, 2008.

40. Mouton JW, Ambrose PG, Canton R et al: Conserving antibiotics for the future: New ways to use old and new drugs from a pharmacokinetic and pharmacodynamic perspective. Drug Resist Updat 14: 107-117, 2011.

41. Reichert JM: Monoclonal antibodies as innovative therapeutics. Curr Pharm Biotechnol 9: 423-430, 2008.

42. Lowy I, Molrine DC, Leav BA, et al: Treatment with monoclonal antibodies against Clostridium difficile toxins. N Engl J Med 362: 197-205, 2010.

43. Lopez EL, Contrini MM, Glatstein E, et al: Safety and pharmacokinetics of urtoxazumab, a humanized monoclonal antibody, against Shiga-like toxin 2 in healthy adults and in pediatric patients infected with Shiga-like toxin-producing Escherichia coli. Antimicrob Agents Chemother 54: 239-243, 2010.

44. Verma A, Schirm M, Arora SK, Thibault P, Logan SM and Ramphal R: Glycosylation of b-type flagellin of Pseudomonas aeruginosa: structural and genetic basis. J Bacteriol 188: 43954403, 2006

45. Shanks KK, Guang W, Kim KC and Lillehoj EP: Interleukin-8 production by human airway epithelial cells in response to Pseudomonas aeruginosa clinical isolates expressing type a or type b flagellins. Clin Vaccine Immunol 17: 1196-1202, 2010.
46. Bannister D, Popovic B, Sridharan S, et al: Epitope mapping and key amino acid identification of anti-CD22 immunotoxin CAT-8015 using hybrid $\beta$-lactamase display. Protein Eng Des Sel 24: 351-360, 2011.

47. Bone RC: Monoclonal antibodies to endotoxin. New allies against sepsis? JAMA 266: 1125-1126, 1991.

48. Bone RC: Why sepsis trials fail. JAMA 276: 565-566, 1996.

49. Holmes D: Buy buy bispecific antibodies. Nat Rev Drug Discov 10: 798-800, 2011

50. Gewirtz AT, Vijay-Kumar M, Brant SR, Duerr RH, Nicolae DL and Cho JH: Dominant-negative TLR5 polymorphism reduces adaptive immune response to flagellin and negatively associates with Crohn's disease. Am J Physiol Gastrointest Liver Physiol 290: G1157-G1163, 2006.

51. Blohmke CJ, Park J, Hirschfeld AF, et al: TLR5 as an antiinflammatory target and modifier gene in cystic fibrosis. J Immunol 185: 7731-7738, 2010.

52. Hawn TR, Wu H, Grossman JM, Hahn BH, Tsao BP and Aderem A: A stop codon polymorphism of Toll-like receptor 5 is associated with resistance to systemic lupus erythematosus. Proc Natl Acad Sci USA 102: 10593-10597, 2005.

53. Nilsson E, Larsson A, Olesen HV, Wejaker PE and Kollberg H: Good effect of IgY against Pseudomonas aeruginosa infections in cystic fibrosis patients. Pediatr Pulmonol 43: 892-899, 2008.

54. Nilsson E, Amini A, Wretlind B and Larsson A: Pseudomonas aeruginosa infections are prevented in cystic fibrosis patients by avian antibodies binding Pseudomonas aeruginosa flagellin. J Chromatogr B Analyt Technol Biomed Life Sci: 856: 75-80, 2007. 